\title{
BMJ Open Quality Effect of a pulmonary nodule fact sheet on patient anxiety and knowledge: a quality improvement initiative
}

Matthew T Koroscil, Mitchell H Bowman, Michael J Morris, Andrew J Skabelund, Andrew M Hersh

To cite: Koroscil MT, Bowman MH, Morris MJ, et al. Effect of a pulmonary nodule fact sheet on patient anxiety and knowledge: a quality improvement initiative. BMJ Open Quality 2018;7:e000437. doi:10.1136/ bmjoq-2018-000437

Received 4 July 2018 Revised 30 July 2018 Accepted 21 August 2018

\section{Check for updates}

(C) Author(s) (or their employer(s)) 2018. Re-use permitted under CC BY-NC. No commercial re-use. See rights and permissions. Published by BMJ.

San Antonio Military Medical Center, Fort Sam Houston, Texas, USA

Correspondence to Dr Matthew T Koroscil; matt.koroscil@gmail.com

\section{ABSTRACT}

Introduction The utilisation of chest CT for the evaluation of pulmonary disorders, including low-dose CT for lung cancer screening, is increasing in the USA. As a result, the discovery of both screening-detected and incidental pulmonary nodules has become more frequent. Despite an overall low risk of malignancy, pulmonary nodules are a common cause of emotional distress among adult patients. Methods We conducted a multi-institutional quality improvement (QI) initiative involving 101 participants to determine the effect of a pulmonary nodule fact sheet on patient knowledge and anxiety. Males and females aged 35 years or older, who had a history of either screeningdetected or incidental solid pulmonary nodule(s) sized 3-8 $\mathrm{mm}$, were included. Prior to an internal medicine or pulmonary medicine clinic visit, participants were given a packet containing a pre-fact sheet survey, a pulmonary nodule fact sheet and a post-fact sheet survey.

Results of 101 patients, 61 (60.4\%) worried about their pulmonary nodule at least once per month with $18(17.8 \%)$ worrying daily. The majority 67/101 (66.3\%) selected chemotherapy, chemotherapy and radiation, or radiation as the best method to cure early-stage lung cancer. Despite ongoing radiographic surveillance, 16/101 (15.8\%) stated they would not be interested in an intervention if lung cancer was diagnosed. Following review of the pulmonary nodule fact sheet, $84 / 101(83.2 \%)$ reported improved anxiety and 96/101 (95.0\%) reported an improved understanding of their health situation. Patient understanding significantly improved from $4.2 / 10.0$ to $8.1 / 10.0(p<0.01)$.

Conclusion The incorporation of a standardised fact sheet for subcentimeter solid pulmonary nodules improves patient understanding and alleviates anxiety. We plan to implement pulmonary nodule fact sheets into the care of our patients with low-risk subcentimeter pulmonary nodules.

\section{INTRODUCTION}

The annual frequency of chest CT imaging continues to increase in the USA, ${ }^{1}$ which is likely a result of increased CT availability and adoption of low-dose CT for lung cancer screening. Between 2006 and 2012, annual chest CT utilisation increased to nearly $2.0 \%$ for all adults and imaging identified a pulmonary nodule on up to $31 \%$ of adults. ${ }^{2}$ As expected, the application of the Fleischner
Society and Lung CT Screening Reporting and Data System (Lung-RADS) recommendations frequently leads to repeat chest CT imaging. ${ }^{3}{ }^{4}$ We have observed that our patients often lack an understanding for the aetiology, malignancy risk and ramification of pulmonary nodules. Despite an overall low incidence of malignancy for subcentimeter nodules, nodule identification is a common cause of emotional distress among patients. ${ }^{5}$ Patients tend to substantially overestimate their risk of lung cancer ${ }^{5}$; however, high-quality communication strategies have been shown to improve patient knowledge about malignancy risk and reduce emotional distress. ${ }^{6}$

In an effort to improve patients' anxiety and understanding, we undertook a quality improvement (QI) initiative that focused on providing an educational fact sheet to our patients with incidental and screening-detected pulmonary nodules. To our knowledge, no study has evaluated the effect of a nodule fact sheet on patient knowledge and anxiety. The goal of the project was to evaluate whether a standardised nodule fact sheet improved patient understanding and anxiety about their nodule(s). The nodule fact sheet was structured to provide easy-to-understand information about the aetiology, malignancy risk and medical consequences of a pulmonary nodule. We expected this intervention would improve patient understanding and decrease patient anxiety as measured by a subjective scale.

\section{METHODS}

A pulmonary nodule fact sheet (figure 1) was developed that contained simple yet pertinent information. It was used in the pulmonary and internal medicine clinics at Brooke Army Medical Center and Wilford Hall Ambulatory Surgery Center in San Antonio, Texas. A pre-fact sheet survey and post-fact sheet survey were then created to evaluate changes 
Patient Copy

\title{
Brooke Army Lung Nodule Factsheet
}

\author{
${ }^{*}$ For solid lung nodules between $3-8 \mathrm{~mm}$ found on CT imaging
}

What is a lung nodule?

A lung nodule is also known as a pulmonary nodule. A lung nodule is small, well-defined area of abnormal lung tissue. They are usually discovered on an X-ray or a CT scan of your chest.

How common are lung nodules?

Lung nodules are very common in our population. Over $25 \%$ of adults older than 65 years old have a pulmonary nodule. Smokers or former smokers have a higher rate of lung nodules when compared to patients who have never smoked.

What causes lung nodules?

The vast majority of lung nodules are "benign", or also known as, non-cancerous. Common causes include "granulomas", which are caused by your immune system and "hamartomas", which are benign areas of normal tissue growth. A very small minority of lung nodules represent cancer.

How does your doctor manage lung nodules?

The majority of lung nodules are safely followed with imaging tests such as CT scans. If the nodule gets smaller or stays a stable size for a certain period of time (generally 1-2 years), the nodule is very unlikely to be cancer and usually does not have to be followed anymore. If the lung nodule is getting bigger with repeat CT scans or has other features that alarm your doctor, a tissue sample may need to be obtained. Getting a tissue sample using needle biopsy or surgery is the only way to diagnose lung cancer. By itself, a CT scan cannot diagnose lung cancer.

Should you be concerned about your lung nodule?

As stated above, lung nodules are very common and most lung nodules are benign (not cancer). In fact, over $99 \%$ of lung nodules less than $5 \mathrm{~mm}$ in diameter (about $1 / 5$ of an inch) are benign. About $95 \%$ of pulmonary nodules that are $5-8 \mathrm{~mm}$ in diameter are benign. Asking questions about your lung nodule with a trusted provider can help improve your stress.

What if my lung nodule is lung cancer?

The goal of following lung nodules with CT scans is to identify patients with early lung cancer. Early stage lung cancer (before it spreads) is best treated with surgery, which requires the surgeon to remove a portion of your lung. You and your doctor should always have an informed discussion before following lung nodules. If you are not willing to undergo surgery or radiation therapy for lung cancer, there may be no benefit to following your lung nodule and this should be discussed with your doctor.

Figure 1 The Brooke Army lung nodule fact sheet.

in patient knowledge and anxiety. We identified adult patients who had a history of either a solitary or multiple solid nodules between 3 and $8 \mathrm{~mm}$ in diameter and chest CT imaging completed within the past twoyears. Both incidental and screening-detected nodules were included. Patients with a history of malignancy, other than non-melanoma skin cancer, and age under 35 were excluded. Patients $<35$ years of age were excluded because the Fleischner Society guidelines for the management of incidental pulmonary nodules do not apply to younger patients and Lung-RADS has been validated only in older patients enrolled in lung cancer screening programmes. Patients with coexistent subsolid or ground glass nodules were also excluded because subsolid nodules have a higher risk of malignancy than solid nodules and ground glass nodules may require prolonged surveillance. ${ }^{78}$ All of this information was obtained by chart review performed by a pulmonary medicine fellow and/or an internal medicine 
resident prior the patient's clinic visit. Candidates for our QI project were given a packet containing documents in the following order: (1) pre-fact sheet survey, (2) pulmonary nodule fact sheet and (3) post-fact sheet survey. While in the clinic waiting room, the patients were asked to complete the surveys in the correct order and return to the clinician or the front desk. If patient answered 'no' to the first question on pre-survey (Are you being followed by a doctor for a lung nodule?), the patient was not included in the analysis. Only completed pre-fact sheet and post-fact sheet surveys were included. Patients were instructed to keep the pulmonary nodule fact sheet for reference after their encounter.

The pre-fact sheet and post-fact sheet surveys were designed to assess patient anxiety and knowledge. A 10-point scale was used to evaluate patient understanding and the improvement of anxiety. Care was made to develop a fact sheet that could be readily understood by a wide population, and the surveys were intended to be short and concise to facilitate better response rates.

The comparison of the pre-fact sheet and post-fact sheet responses were used to determine the effect of the intervention. The post-fact sheet survey also contained yes or no questions about whether the fact sheet improved understanding and anxiety.

The protocol for the administration of the surveys and fact sheets was determined as QI by our institution's institutional review board. Since the aim of the initiative was to improve patient understanding about pulmonary nodules and reduce stress without the intention to create generalisable knowledge, our project received a QI determination. There were no concerning ethical aspects of implementing and studying the effect of pulmonary nodule fact sheet on patient anxiety and knowledge.

We compared central tendency and frequency for using the two-sample t-test for continuous values, and the $\chi^{2}$ test or the Fisher's exact test when sample size was small.

\section{RESULTS}

Survey data were collected from 101 participants. All participants completed the surveys prior to an internal medicine or pulmonary clinic encounter. Of the 101 surveys, $63(62.4 \%)$ were from the internal medicine clinic and $38(37.6 \%)$ were from the pulmonary clinic. Initially, the packet containing the questionnaires and the fact sheet was placed into the appropriate patient folder without specific instruction to the front desk or the participant. We observed a $10 \%$ response rate likely due to the poor communication between the front desk and patients. We therefore modified our intervention by instructing our front desks to inform participants about the packet, and we added a highlighted section on the questionnaires that reminded patients to return the documents to the front desk. This updated protocol improved our participant response rate $>70 \%$.
Table 1 Percentage of patients $(n=101)$ who perceived improvement in anxiety and understanding

\begin{tabular}{ll}
\hline & Per cent perceived improvement \\
\hline Anxiety & 83.2 \\
Understanding & 95.0 \\
\hline
\end{tabular}

\section{Anxiety}

From the pre-fact sheet survey, 61/101 (60.4\%) patients noted worries about their pulmonary nodule at least once per month with 21/101 (20.8\%) worrying at least daily. Only 24/101 (23.8\%) never worried about their pulmonary nodule. The participants rated their anxiety using a 10-point scale with 10 indicating maximal anxiety. Patients had a mean pre-fact sheet anxiety score of 4.9/10.0. Following review of the pulmonary nodule fact sheet, $84 / 101(83.2 \%)$ reported improved anxiety (table 1) and rated anxiety improvement using a 10-point scale to a mean 6.7/10.0 with 1.0 representing no improvement and 10.0 indicating maximal improvement. There was no significant difference in terms of anxiety between the internal medicine and pulmonary clinic patients.

\section{Knowledge}

Using a 10-point scale, participants rated their understanding of pulmonary nodules to a mean of $4.2 / 10.0$ with 1.0 representing poor understanding and 10.0 representing great understanding. Again, there was not a significant difference between the responses of patients surveyed in the internal medicine versus the pulmonary clinic. Also, 34 of 101 (33.6\%) patients correctly estimated the prevalence of pulmonary nodules in adult patients. A minority of participants $34 / 101$ (33.6\%) selected lung surgery as the best method to cure early-stage lung cancer. The majority $67 / 101$ (66.3\%) selected chemotherapy, chemotherapy and radiation, or radiation as preferred method to cure early-stage lung cancer. Most patients $(85 / 101 ; 84.2 \%)$ would consider surgery or radiation if a diagnosis of lung cancer was made, but 16/101 (15.8\%) stated they would not be willing to undergo intervention if lung cancer was diagnosed. The majority of the patients unwilling to undergo surgery or radiation were internal

\section{Patient Understanding}

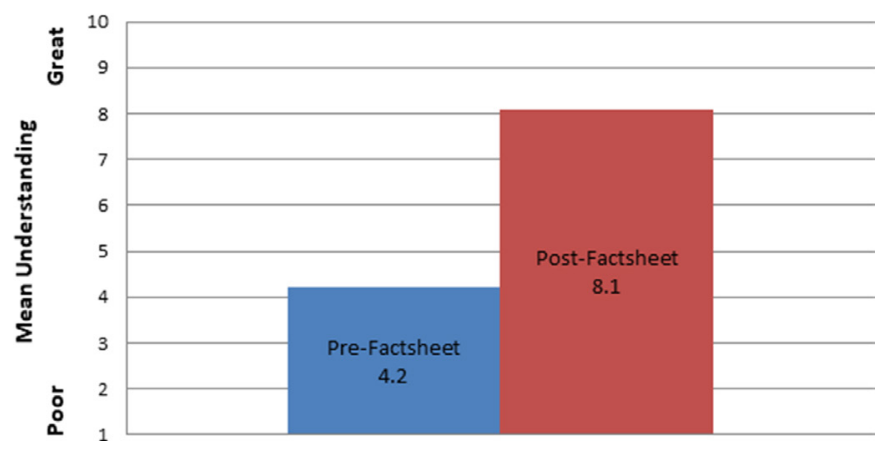

Figure 2 The mean patient understanding pre-fact sheet and post-fact sheet $(p<0.01)$. 
medicine patients 13/63 (20.6\%) versus 3/38 (7.9\%) of pulmonary clinic patients. Following the intervention, patient understanding significantly improved to $8.1 / 10.0$ $(p<0.01)$ (figure 2$)$ and the majority 96/101 $(95.0 \%)$ of participants reported an improvement of understanding about their health situation.

\section{DISCUSSION}

In this multi-institutional QI initiative, the incorporation of a pulmonary nodule fact sheet improved patient understanding and anxiety. Over $60 \%$ of patients worried at least once a month about the status of their pulmonary nodule(s) prior to being evaluated by a provider. This is the first study to show the added benefit of a pulmonary nodule fact sheet on both general internal medicine and pulmonary medicine patients.

With the advent of lung cancer screening and increased chest CT utilisation, the amount of screening-detected and incidental pulmonary nodules is increasing. Whether screening-detected or incidental, most nodules are subcentimeter in size and have an overall low risk of malignancy. The fact sheet quoted a $5 \%$ or less risk of malignancy among solid nodules $<8 \mathrm{~mm}$ in diameter. This risk of malignancy is consistent with data on screening-detected nodules and incidental nodules. ${ }^{9}{ }^{10}$ Multiple studies have shown that patients tend to receive inadequate information about their pulmonary nodule. ${ }^{6} 1112$ A good communication strategy to help improve patient understanding reduces distress. Following review of our pulmonary nodule fact sheet, there was a statistically significant improvement in the patients' perception of understanding. Additionally, 96/101 (95\%) of patients reported an improved understanding of their health situation. Enhanced patient education has been associated with improved patient satisfaction and possibly clinical outcomes among diverse disease processes. ${ }^{13-15}$ We expect that our patients' improved understanding of pulmonary nodules would translate to better satisfaction and thus quality of care.

The degree of anxiety identified in our study is consistent with previous studies, which report an overestimation of risk of lung cancer among Veteran Affairs patients with incidental nodules. ${ }^{56}$ The improvement in self-reported anxiety in our study may be secondary to the fact sheet's discussion on the commonality of subcentimeter pulmonary nodules and the overall low malignancy risk. The risk of malignancy is important to patients and often not included in initial discussion. ${ }^{12}$ Our fact sheet's effect on patient anxiety was not unexpected as several studies have shown patient education can reduce anxiety, a likely result of improved knowledge. ${ }^{16} 17$ We believe that the improved understanding of pulmonary nodules helped make our patients' perceived risk of lung cancer more congruent with the actual risk of lung cancer and therefore reduced anxiety.

Only $33.7 \%$ of patients were able to identify the prevalence of pulmonary nodules or determine the best modality to treat early-stage lung cancer. The majority of our participants $(40.6 \%)$ believed chemotherapy and radiation was the treatment of choice for early-stage lung cancer. Importantly, $15.8 \%$ of the patients stated that they would not be interested in surgery or radiation if lung cancer was diagnosed. After high-quality communication and family discussion, it is possible that many of these patients would reconsider if curative intervention was possible; however, recognition of these initial preferences is an important consideration when clinicians decide how aggressively to pursue nodule follow-up. Our results reiterate the importance of patient-centred communication as there likely is a minority of patients who would refuse any intervention based on personal preference. Repeat imaging in such individuals would not be beneficial and would add to overall healthcare costs with increased radiation exposure.

There are several strengths of our project. We included completed questionnaires by $>100$ patients in diverse clinical settings. We had a systematic approach to selecting patients so only low-risk pulmonary nodules were included. This helped prevent the distribution of the nodule fact sheet to high-risk patients with larger pulmonary nodules and history of malignancy. Our patient population is mostly an older cohort of military retirees and their spouses; however, the amount of emotional distress caused by pulmonary nodules in our population appeared similar to prior studies.

There are several limitations to this quality improvement project. First, we only included surveys in patients who answered 'yes' to the first question on pre-fact sheet survey (Are you being followed by a doctor for a lung nodule?). As a result, numerous patients with a history of subcentimeter pulmonary nodules were excluded. Second, we used a non-validated scale for measuring anxiety and knowledge. Third, our fact sheet only assessed the short-term knowledge and anxiety. Therefore, it is unclear if the benefit of a nodule fact sheet would be sustained long term. Lastly, we are unable to assess if this improved knowledge leads to different patient choices.

\section{CONCLUSION}

As the general use of chest CT, including lung cancer screening, continues to grow, the risk of causing undue anxiety becomes evermore present. The incorporation of a standardised fact sheet for subcentimeter solid pulmonary nodules appears to improve patient understanding and alleviate anxiety. This intervention improves the care of our patients and, in combination with simple communication strategies, may become the standard of care. Future studies are needed to determine the optimal timing and content of these fact sheets. The next step of this QI initiative will be to refine and expand on the lung nodule fact sheet, explore additional modalities for improving patient education and reducing anxiety, as well as testing the durability of these improvements over time. 
Contributors MTK planned, conducted and created the manuscript. MTK is the guarantor. MHB helped conduct and create the manuscript. AJS helped plan the project. MJM and AMH helped create the manuscript.

Funding The authors have not declared a specific grant for this research from any funding agency in the public, commercial or not-for-profit sectors.

Disclaimer The views expressed herein are those of the authors and do not reflect the official policy or position of the Department of the Army, the Department of the Air Force, the Department of Defense, or the US Government.

Competing interests None declared.

Patient consent Not required.

Provenance and peer review Not commissioned; externally peer reviewed.

Open access This is an open access article distributed in accordance with the Creative Commons Attribution Non Commercial (CC BY-NC 4.0) license, which permits others to distribute, remix, adapt, build upon this work non-commercially, and license their derivative works on different terms, provided the original work is properly cited, appropriate credit is given, any changes made indicated, and the use is non-commercial. See: http://creativecommons.org/licenses/by-nc/4.0/.

\section{REFERENCES}

1. Smith-Bindman R, Miglioretti DL, Johnson E, et al. Use of diagnostic imaging studies and associated radiation exposure for patients enrolled in large integrated health care systems, 1996-2010. JAMA 2012;307:2400-9.

2. Gould MK, Tang T, Liu IL, et al. Recent trends in the identification of incidental pulmonary nodules. Am J Respir Crit Care Med 2015;192:1208-14.

3. MacMahon H, Naidich DP, Goo JM, et al. Guidelines for management of incidental pulmonary nodules detected on CT images: from the Fleischner society 2017. Radiology 2017;284:228-43.

4. Pinsky PF, Gierada DS, Black W, et al. Performance of lung-RADS in the National Lung Screening Trial: a retrospective assessment. Ann Intern Med 2015;162:485-91.
5. Slatore CG, Wiener RS, Golden SE, et al. Longitudinal assessment of distress among veterans with incidental pulmonary nodules. Ann Am Thorac Soc 2016;13:1983-91.

6. Slatore CG, Golden SE, Ganzini L, et al. Distress and patientcentered communication among veterans with incidental (not screendetected) pulmonary nodules. A cohort study. Ann Am Thorac Soc 2015:12:184-92.

7. Naidich DP, Bankier AA, MacMahon $\mathrm{H}$, et al. Recommendations for the management of subsolid pulmonary nodules detected at CT: a statement from the Fleischner Society. Radiology 2013;266:304-17.

8. Henschke Cl, Yankelevitz DF, Mirtcheva R, et al. CT screening for lung cancer: frequency and significance of part-solid and nonsolid nodules. AJR Am J Roentgenol 2002;178:1053-7.

9. McWilliams A, Tammemagi MC, Mayo JR, et al. Probability of cancer in pulmonary nodules detected on first screening CT. N Engl J Med 2013;369:910-9.

10. MacMahon H, Naidich DP, Goo JM, et al. Guidelines for management of incidental pulmonary nodules detected on CT images: from the Fleischner Society 2017. Radiology 2017;284:228-43.

11. Slatore CG, Press N, Au DH, et al. What the heck is a "nodule"? A qualitative study of veterans with pulmonary nodules. Ann Am Thorac Soc 2013;10:330-5.

12. Wiener RS, Gould MK, Woloshin S, et al. What do you mean, a spot?: A qualitative analysis of patients' reactions to discussions with their physicians about pulmonary nodules. Chest 2013;143:672-7.

13. Medrano Martínez V, Callejo-Domínguez JM, Beltrán-Lasco I, et al. Migraine education brochures and patient-perceived satisfaction. Neurologia 2015;30:472-8.

14. Vinicor F, Cohen SJ, Mazzuca SA, et al. DIABEDS: a randomized trial of the effects of physician and/or patient education on diabetes patient outcomes. J Chronic Dis 1987;40:345-56.

15. Runge C, Lecheler J, Horn M, et al. Outcomes of a Web-based patient education program for asthmatic children and adolescents. Chest 2006;129:581-93.

16. Cetkin HE, Tuna A. How does health education given to lung cancer patients before thoracotomy affect pain, anxiety, and respiratory functions? J Cancer Educ 2018 (Epub ahead of print 18 Jul 2018).

17. Habibzadeh H, Milan ZD, Radfar M, et al. Effects of peer-facilitated, video-based and combined peer-and-video education on anxiety among patients undergoing coronary angiography: randomised controlled trial. Sultan Qaboos Univ Med J 2018;18:61. 